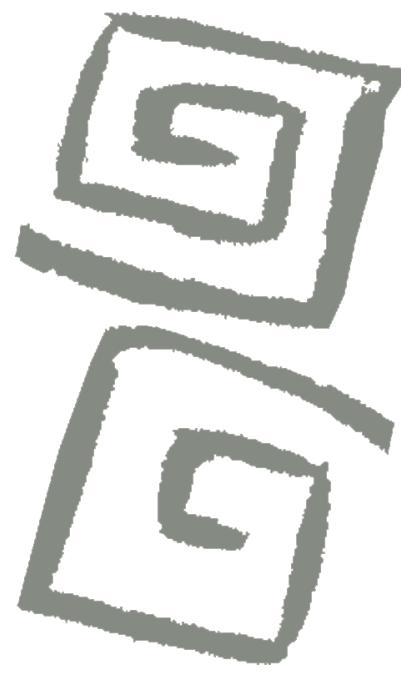

\title{
El perfil de usuarios de benzodiazepinas en servicios de atención primaria de la salud de la ciudad de Chapecó, Santa Catarina, Brasil
}

\author{
Profile of benzodiazepine users attending primary \\ healthcare services in the city of Chapecó, Santa \\ Catarina, Brazil
}

${ }^{1}$ Autor de correspondencia. Doctora en Salud Colectiva, área Epidemiología. Profesora Adjunta, Universidade Federal da Fronteira Sul, campus Chapecó. Santa Catarina, Brasil. $\triangle$ (iD)

${ }^{2}$ Doctora en Enfermería. Profesora Adjunta, Universidade Federal da Fronteira Sul, campus Chapecó. Santa Catarina, Brasil. $\bowtie$ iD

${ }^{3}$ Académica. Universidade Federal da Fronteira Sul, campus Chapecó. Santa Catarina, Brasil. $\bowtie$ (iD)

${ }^{4}$ Académica. Universidade Federal da Fronteira Sul, campus Chapecó. Santa Catarina, Brasil. $\square$ (iD)

${ }^{5}$ Académica. Universidade Federal da Fronteira Sul, campus Chapecó. Santa Catarina, Brasil. $\square$ (iD)

${ }^{6}$ Doctor en Salud Colectiva. Profesor Adjunto, Universidade Federal da Fronteira Sul, campus Chapecó. Santa Catarina, Brasil. $\square$ iD
Jane Kelly Oliveira Friestino' , Adriana Remião Luzardo², Érika Zachi Gralak³, Letícia Scherer Gass ${ }^{4}$, Patrícia Gonçalves Zambrano Guimarães 5 , Paulo Roberto Barbato ${ }^{6}$

RESUMEN La atención primaria de la salud es un punto transversal dentro de la Red de Atención Psicosocial, y atiende las demandas relacionadas con el sufrimiento mental. Si bien los medicamentos representan la principal opción de tratamiento, el uso prolongado de benzodiazepinas puede desarrollar tolerancia y dependencia. El objetivo es analizar la frecuencia y las características de los usuarios de benzodiazepinas en los servicios de atención primaria de la salud, a través de un estudio transversal realizado en la ciudad de Chapecó, estado de Santa Catarina, un municipio del sur de Brasil. Se verificó un elevado número de prescripciones proporcionales entre mujeres, adultos y ancianos y un predominio de personas de sexo femenino $(80 \%)$, color de piel blanca $(67,9 \%)$, casadas $(50,7 \%)$, con una media de edad de 56 años, que señala la necesidad de diseñar e implementar cambios en los procedimientos de prescripción y dispendio de benzodiazepinas.

PALABRAS CLAVES Medicalización; Atención Primaria de Salud; Salud Mental; Brasil.

\begin{abstract}
Primary Health Care is a transversal point in the Psychosocial Care Network, responsible for attending to demands related to mental suffering. Although medication represents the main treatment option, the prolonged use of benzodiazepines may lead to tolerance and consequent dependence. The objective of this study is to analyze the frequency and characteristics of benzodiazepine users attending primary healthcare services, through a cross-sectional study conducted in Chapecó, Santa Catarina, a municipality in southern Brazil. Higher proportional prescription was observed in adult and elderly women, with predominance among individuals who were women $(80 \%)$, white $(67.9 \%)$, and married $(50.7 \%)$, with a median age of 56 years. These findings suggest a need to plan and implement changes in the procedures for prescribing and administering benzodiazepines.
\end{abstract}

KEY WORDS Medicalization; Primary Health Care; Mental Health; Brazil. 


\section{INTRODUCCIÓN}

En la década de 1970, en un escenario nacional de redemocratización y de lucha contra la violencia institucional, se iniciaron los primeros movimientos de reconstrucción de la asistencia psiquiátrica en Brasil( ${ }^{(1)}$. En ese período, se produjo una importante discusión sobre los derechos de los usuarios de los servicios públicos de salud, promovida por el movimiento sanitarista y antimanicomial, que repercutió en la salud pública desde entonces hasta la actualidad ${ }^{(2)}$.

El movimiento sanitarista promovía un sistema de salud con capacidad de ofrecer cuidados preventivos y curativos como un derecho de toda la ciudadanía, a través de un sistema integrado, con descentralización de la gestión y control social de las acciones en salud ${ }^{(3)}$. Sus propuestas se oponían al modelo biomédico predominante hasta entonces, el cual se centraba solo en tratar la enfermedad y los síntomas ya instalados, sin considerar el contexto psicosocial en el que el usuario estaba inserto ni la prevención de enfermedades o promoción de la salud para la población ${ }^{(4)}$.

Después de años de desarrollo y estructuración del movimiento, en 1990, con la Ley Federal 8080 se estableció el Sistema Único de Salud (SUS), cuyos principios basados en la universalización, la equidad y la integralidad de la salud permitieron la participación popular, la descentralización y la división de los servicios en niveles de atención ${ }^{(5)}$, garantizando el cuidado organizado y la mejora de la salud de la población.

En lo concerniente a la atención del sufrimiento mental, en 2001, se sancionó la Ley Federal 10216 sustentada en los principios de la Reforma Psiquiátrica, que puso fin a un período retrógrado de asistencia a la salud mental. A partir de entonces se reestructuró el modelo asistencial en salud mental y se establecieron los derechos de los usuarios, garantizando el cuidado integral y su reinserción en la sociedad ${ }^{(6)}$. Así, por medio de la consolidación del SUS y la Reforma Psiquiátrica, se buscó la efectivización de un nuevo modelo de atención a la salud, basado en la integralidad del cuidado al paciente, que posibilitó que los servicios psiquiátricos comenzaran a recomendar la introducción gradual de las personas con sufrimiento mental en la sociedad, a través de acciones de prevención, promoción, tratamiento y rehabilitación ${ }^{(6,7)}$.

Una de las conquistas de la Reforma Psiquiátrica fue que las acciones en salud mental se descentralizaran hacia los territorios, lo cual exigió una mejora constante de ese modelo ${ }^{(8)}$. Actualmente, el cuidado en salud mental en atención primaria se configura como una acción estratégica, dado que es cada vez más común la demanda del servicio por parte de usuarios con sufrimiento psíquico $^{(9)}$. Por lo tanto, capacitar a los profesionales para lidiar con esa problemática es un ejercicio esencial.

Aunque la experiencia brasileña en atención a la salud mental aún pueda ser considerada incipiente, hay países en América Latina, como Ecuador, que recién en la última década implementaron estrategias dirigidas a los cuidados referentes al sufrimiento mental y que aun hoy enfrentan dificultades, tanto presupuestarias como de falta de profesionales calificados para ese abordaje en atención primaria ${ }^{(10)}$, dado que los rasgos del modelo biomédico aún están presentes en los diversos sectores de la salud, tanto en países de América Latina, como en Brasil( ${ }^{(9)}$.

En general, las personas con sufrimiento mental encuentran en los servicios de salud una atención esencialmente farmacológica que, a través de la terapia medicamentosa, apunta a eliminar el problema instantáneamente. Sin embargo, se podrían incorporar otras posibilidades a las prácticas de cuidado de la salud, como las descritas por la Política Nacional de Prácticas Integrales y Complementarias, que consideran la subjetividad y la singularidad de los sujetos ${ }^{(11)}$.

La atención primaria, especialmente a través de la Estrategia de Salud Familiar, orienta el modelo asistencial a partir de una perspectiva psicosocial, que incluye el trabajo continuo en el territorio y la formación de vínculos, propiciando el contacto directo con las demandas en salud mental ${ }^{(11)}$. Por esta razón, la atención 
primaria pasa a ser un punto transversal en la Red de Atención Psicosocial, y es responsable de atender demandas relacionadas con el sufrimiento mental, que incluyen trastornos de ansiedad, del sueño y del humor.

La gran demanda de usuarios de atención primaria, relacionada con el estrés psicológico, sumada a la prescripción poco controlada de las benzodiazepinas por parte de las y los profesionales que actúan en atención primaria, la distribución gratuita, y también la falta de formación de los equipos de salud para garantizar la receptividad adecuada de la persona con sufrimiento mental ${ }^{(12)}$ contribuyen, junto a otras causas, al dispendio indiscriminado de medicamentos psicotrópicos y la consiguiente medicalización de diferentes perfiles de usuarios.

Por lo tanto, resulta relevante conocer la tradición de los medicamentos psicotrópicos en la atención primaria de la salud y entender cómo, a lo largo del tiempo, esos medicamentos, sobre todo la clase de ansiolíticos como las benzodiazepinas, contribuyeron a la medicalización de personas con sufrimiento mental y su consecuente dependencia por esas sustancias.

Durante la década de 1950, el desarrollo y la introducción de las benzodiazepinas en el mercado farmacéutico se consideró un hito en la terapéutica psiquiátrica, presentado como una alternativa segura, eficaz y con menos efectos adversos en el tratamiento de enfermedades relacionadas con el sistema nervioso central, por lo que fueron ampliamente prescritos y se tornaron muy populares $^{(7)}$.

A partir de 1960, con el lanzamiento del clonazepam en el mercado comercial, se abrieron las puertas para que, a partir de cambios estructurales en la molécula original, se pudieran sintetizar diversos derivados de esas benzodiazepinas, como el diazepam, en 1963, y el lorazepam, en $1970^{(13)}$, utilizados hasta la actualidad.

La tolerancia y la dependencia potenciales a las benzodiazepinas, debido al uso prolongado, se debe, sobre todo, a la infrarregulación de los receptores y al aumento de la tasa de metabolismo del fármaco debido a la reiterada exposición. O sea, la estimulación prolongada por esta clase de medicamentos no solo promueve la dependencia fisiológica derivada de los efectos tranquilizantes del uso de los fármacos, sino que también conlleva a la insensibilidad gradual de los receptores, provocando que el organismo demande dosis cada vez más altas. Por otra parte, ciertos estudios muestran que la gravedad de los síntomas de abstinencia se relacionan directamente con la característica del fármaco ${ }^{(14)}$.

Además del efecto proporcionado por la acción de las drogas psicotrópicas, el mercado farmacéutico también contribuye a que los medicamentos se consideren como la primera opción, teniendo en cuenta la facilidad de dispendio y la variedad de medicamentos disponibles. Sin embargo, a pesar de ser una clase menos tóxica que los barbitúricos, también causan innumerables efectos adversos, además de la ya conocida acción acumulativa provocada por la administración de múltiples dosis ${ }^{(14)}$.

A lo largo de los últimos 20 años, con el aumento del consumo de benzodiazepinas en Brasil y en el mundo, se realizaron diversos estudios sobre el tema ${ }^{(15)}$ que caracterizan el uso de los fármacos ${ }^{(16,17)}$, analizan las prescripciones ${ }^{(18)}$ y evalúan la orientación médica respecto de los fármacos ${ }^{(19)}$. Tales investigaciones cuestionan la prescripción de medicamentos como terapia única y la necesidad de seguimiento y cuidados que el usuario de esta clase de fármacos requiere a lo largo del tiempo, respetando la posibilidad de reducir las dosis y la adopción de medidas integrales que actúen conjuntamente con el fármaco en el alivio del sufrimiento mental.

Conocer las particularidades locales de la relación de la población con el uso de benzodiazepinas resulta importante, dado que aporta a la comprensión de la compleja realidad de los procesos de medicalización ${ }^{(20)}$, y brinda la posibilidad de definir acciones más asertivas respecto al cuidado de las personas con sufrimiento mental.

El presente estudio forma parte de un proyecto mayor, titulado Sofrimento mental na Atenção Básica: profissionais, diagnóstico e tratamento em um município da região oeste 
de Santa Catarina. Su objetivo es analizar la frecuencia y las características de los usuarios de benzodiazepinas en atención primaria, considerando las particularidades locales.

\section{MÉTODOS}

Se trata de un estudio transversal, realizado en el municipio de Chapecó, estado de Santa Catarina, con datos recolectados en la atención primaria, a partir de registros de consultas atendidas en el año de 2016.

Se incluyeron en el estudio aquellos usuarios de servicios de atención primaria que recibieron prescripciones de benzodiazepinas (clonazepam, diazepam y lorazepam) realizadas por médicos de familia y médicos generalistas. Se excluyeron aquellos usuarios que recibieron prescripciones de benzodiazepinas pero que podrían ser utilizadas para casos de trastornos neurológicos, además de los que recibieron prescripciones de benzodiazepinas no incluidas en los listados de la Relación Municipal de Medicamentos Esenciales (REMUME) del municipio estudiado.

\section{Población y muestra}

Chapecó es una ciudad del estado de Santa Catarina, Brasil, que de acuerdo con el censo poseía, en 2010, 183.530 habitantes, de los cuales 90.626 eran varones y 92.904 mujeres, y una población estimada, en 2016, de 209.553 habitantes, con un índice de crecimiento demográfico del 4,89\%. En el momento de la investigación, la ciudad poseía 27 centros de salud familiar, y contaba con 46 equipos de Estrategia de Salud Familiar, con una cobertura del $77,12 \%$ de la población atendida en los servicios de atención primaria del SUS, distribuidos entre la zona rural y la zona urbana de la ciudad ${ }^{(21)}$.

Para determinar los centros de salud familiar a ser incluidos en la investigación, fue necesario, en primer lugar, conocer las prescripciones de los fármacos psicotrópicos generadas por médicos actuantes en los servicios de atención primaria del municipio. Para ello, se realizó un relevamiento de los siete principales psicotrópicos dispensados por el SUS en las seis farmacias del municipio, desde el 1 de marzo al 31 de mayo de 2016, estableciendo un período de tres meses para que no se perdieran individuos y no se reiteraran las prescripciones. Este proceso de muestreo se realizó junto con la dirección de asistencia farmacéutica del municipio. Se generaron informes de prescripciones de los siguientes fármacos: clonazepam $5 \mathrm{mg}$, clonazepam $10 \mathrm{mg}$, diazepam $5 \mathrm{mg}$, diazepam $10 \mathrm{mg}$, amitriptilina $25 \mathrm{mg}$, citalopram $20 \mathrm{mg}$ y fluoxetina $20 \mathrm{mg}$. Estos informes se agruparon de acuerdo al centro de salud familiar desde donde se había prescripto el fármaco y, a partir de los números obtenidos, se realizó un cálculo para determinar la proporción de dispendio con relación a la población adscrita en cada centro de salud.

A partir de esta muestra intencional, se identificaron dos centros de salud familiar con mayor proporción de prescripciones individuales (nominados en este estudio como CSF1 Zona Urbana y CSF2 Zona Rural), y dos centros de salud familiar con la menor proporción de prescripciones (CSF3 Zona Rural y CSF4 Zona Urbana). Estos cuatro centros de salud familiar se configuraron como el locus para la investigación mayor, cuyo objetivo fue conocer cómo se abordaba el tema sufrimiento mental en los servicios de atención primaria del municipio.

\section{Recolección de datos}

Entre marzo y mayo de 2016, se realizó la recolección de datos a partir de la historia clínica electrónica de cada paciente. Se cargó la información en un formulario propio de recolección de datos y se conformó una base de datos que fue procesada y almacenada en planillas de OpenOffice ${ }^{\oplus}$.

En las historias clínicas de los pacientes se analizaron y observaron las siguientes variables: sexo, edad, domicilio, raza, escolaridad, ocupación, benzodiazepinas utilizadas, 
duración del tratamiento y demanda/diagnóstico registrado. Se incluyeron las historias clínicas de aquellos usuarios que tuvieran dispendio en los tres meses descritos en el método de la investigación, y que no fueran atendidos por el Centro de Atención Psicosocial (CAPS), servicio especializado de salud mental.

\section{Análisis de los datos}

El análisis estadístico sobre el conjunto de datos cuantitativos se efectuó con el software Stata for Windows. Se realizó un análisis de frecuencias, análisis de variancia, y se establecieron comparaciones entre las variables descriptivas de las diferentes unidades estudiadas.

\section{Aspectos éticos}

El estudio fue aprobado por el Comité de Ética en Investigación en Seres Humanos de la Universidad Federal de la Fronteira Sul, bajo el parecer No 1.647.056, de 2016 (CAAE: 56468816.5.0000.5564)

Tanto el acceso a las historias clínicas electrónicos del Sistema Único de Salud, como la recolección de esos datos para fines de investigación fue explicitada y autorizada en los términos del consentimiento libre e informado firmado por los participantes.

\section{RESULTADOS}

En la investigación se incluyeron los datos de las historias clínicas de 134 usuarios de la red municipal de salud que tenían prescripción de benzodiazepinas en el período de marzo a mayo de 2016, oriundos de las cuatro unidades de salud analizadas. La media de edad encontrada fue de 55,4 años (desviación estándar 15,5), dentro de un rango de 17 a 92 años. La mediana de la distribución etaria fue de 56 años. La proximidad de la media y mediana de edad indican normalidad en la distribución.
La Tabla 1 presenta el número y el porcentaje de usuarios de la red municipal de salud con prescripción de benzodiazepinas, según las variables seleccionadas. El centro de salud que prescribió más benzodiazepinas se encontraba en la zona urbana $(73,1 \%)$ y rural $(21,6 \%)$. Hubo un predominio del sexo femenino (que constituyeron el $79,8 \%$ del total de participantes), de color de piel blanca $(67,9 \%)$ y estado civil casada/o (50,7\%). A pesar del

Tabla 1. Número y porcentaje de usuarios de la red municipal de salud con prescripción de benzodiazepinas, según variables seleccionadas. Chapecó, Santa Catarina, Brasil, 2016.

\begin{tabular}{|c|c|c|}
\hline Variable & $\mathbf{n}$ & $\%$ \\
\hline Centros de salud & 134 & 100,0 \\
\hline CSF 1 - Zona Urbana & 98 & 71,3 \\
\hline CSF 2 - Zona Rural & 29 & 21,6 \\
\hline CSF 3 - Zona Rural & 4 & 3,0 \\
\hline CSF 4 - Zona Urbana & 3 & 2,3 \\
\hline Droga & 134 & 100,0 \\
\hline Clonazepam & 73 & 54,5 \\
\hline Diazepam & 60 & 44,8 \\
\hline Lorazepam & 1 & 0,7 \\
\hline Sexo & 134 & 100,0 \\
\hline Masculino & 27 & 20,2 \\
\hline Femenino & 107 & 79,8 \\
\hline Franja etaria & 134 & 100,0 \\
\hline$<50$ años & 45 & 33,6 \\
\hline 50-59 años & 37 & 27,6 \\
\hline 60-69 años & 31 & 23,1 \\
\hline$>69$ años & 21 & 15,7 \\
\hline Color de piel & 134 & 100,0 \\
\hline Blanca & 91 & 67,9 \\
\hline Parda/Negra & 43 & 32,1 \\
\hline Estado civil & 134 & 100,0 \\
\hline Casada/0 & 68 & 50,7 \\
\hline Otros & 12 & 9,0 \\
\hline Sin información & 54 & 40,3 \\
\hline Escolaridad & 134 & 100,0 \\
\hline Enseñanza superior/media & 11 & 8,2 \\
\hline Enseñanza primaria & 58 & 43,3 \\
\hline Alfabetizada/0 & 20 & 14,9 \\
\hline Analfabeta/0 & 10 & 7,5 \\
\hline Sin información & 35 & 26,1 \\
\hline Situación de ocupación & 134 & 100,0 \\
\hline Trabajador & 28 & 20,9 \\
\hline Jubilada/0 & 47 & 35,0 \\
\hline Desempleada/o & 9 & 6,7 \\
\hline No trabaja & 12 & 9,0 \\
\hline Sin información & 38 & 28,4 \\
\hline
\end{tabular}

Fuente: Elaboración propia. 
Tabla 2. Prevalencia del uso de benzodiazepinas de acuerdo a la localización de los centros de salud familiar. Chapecó, Santa Catarina, Brasil, 2016.

\begin{tabular}{|c|c|c|c|c|c|}
\hline \multirow{2}{*}{ Variable } & \multicolumn{2}{|c|}{$\begin{array}{c}\text { Centros de salud } \\
\text { familiar en zona rural }\end{array}$} & \multicolumn{2}{|c|}{$\begin{array}{l}\text { Centros de salud } \\
\text { familiar en zona urbana }\end{array}$} & \multirow{2}{*}{ Valor de $p$} \\
\hline & $\%$ & IC 95\% & $\%$ & IC $95 \%$ & \\
\hline \multicolumn{6}{|l|}{ Droga } \\
\hline Clonazepam & 51,5 & $33,5-69,5$ & 55,4 & $45,6-65,3$ & \multirow{3}{*}{0,208} \\
\hline Diazepam & 45,4 & $27,5-63,4$ & 44,5 & $34,7-54,4$ & \\
\hline Lorazepam & 3,0 & $-3,1-9,2$ & - & - & \\
\hline \multicolumn{6}{|l|}{ Sexo } \\
\hline Masculino & 21,2 & $6,5-35,9$ & 19,8 & $11,9-27,7$ & \multirow{2}{*}{0,861} \\
\hline Femenino & 78,8 & $64,1-93,5$ & 80,2 & $72,3-88,1$ & \\
\hline \multicolumn{6}{|l|}{ Franja etaria } \\
\hline$<50$ años & 45,4 & $27,5-63,4$ & 29,7 & $20,6-38,8$ & \multirow{4}{*}{0,348} \\
\hline 50-59 años & 24,2 & $8,8-39,7$ & 28,7 & $19,7-37,7$ & \\
\hline 60-69 años & 21,2 & $6,5-35,9$ & 23,8 & $15,3-32,2$ & \\
\hline$>69$ años & 9,1 & $-1,3-19,4$ & 17,8 & $10,2-25,4$ & \\
\hline \multicolumn{6}{|l|}{ Color de piel } \\
\hline Blanca & 63,6 & $46,3-80,9$ & 69,3 & $60,1-78,4$ & \multirow{2}{*}{0,545} \\
\hline Parda/negra & 36,4 & $19,0-53,7$ & 30,7 & $21,5-39,8$ & \\
\hline \multicolumn{6}{|l|}{ Estado civil } \\
\hline Casada/o & 81,8 & $67,9-95,7$ & 40,6 & $30,8-50,3$ & \multirow{3}{*}{$<0,001$} \\
\hline Otros & 12,1 & $0,4-23,9$ & 7,9 & $2,6-13,3$ & \\
\hline Sin información & 6,1 & $-2,5-14,6$ & 51,5 & $41,6-61,4$ & \\
\hline \multicolumn{6}{|l|}{ Escolaridad } \\
\hline Enseñanza superior/media & 9,1 & $-1,3-19,4$ & 7,9 & $2,6-13,3$ & \multirow{5}{*}{$<0,001$} \\
\hline Enseñanza primaria & 33,3 & $16,3-50,3$ & 46,5 & $36,6-56,4$ & \\
\hline Alfabetizada/0 & 42,4 & $24,6-60,2$ & 5,9 & $1,2-10,6$ & \\
\hline Analfabeta/0 & 9,1 & $-1,3-19,4$ & 6,9 & $1,9-12,0$ & \\
\hline Sin información & 6,1 & $-2,5-14,6$ & 32,7 & $23,4-42,0$ & \\
\hline \multicolumn{6}{|l|}{ Situación de ocupación } \\
\hline Trabajador & 24,2 & $8,8-39,7$ & 19,8 & $11,9-27,7$ & \multirow{5}{*}{0,093} \\
\hline Jubilada/0 & 45,4 & $27,5-63,4$ & 31,7 & $22,4-40,9$ & \\
\hline Desempleada/0 & 12,1 & $0,4-23,9$ & 4,9 & $0,6-9,2$ & \\
\hline No trabaja & 6,1 & $-2,5-14,6$ & 9,9 & $4,0-15,8$ & \\
\hline Sin información & 12,1 & $0,4-23,9$ & 33,7 & $24,3-43,0$ & \\
\hline
\end{tabular}

Fuente: Elaboración propia.

IC 95\% = Intervalo de confianza 95\%.

porcentaje de historias clínicas sin respuesta, se observó un predominio de baja escolaridad y, en cuanto a la ocupación, el mayor porcentaje fue de personas jubiladas $(35,0 \%)$.

La Tabla 2 muestra los datos de prevalencia del uso de benzodiazepinas de acuerdo con las variables de exposición, según la ubicación de los centros de salud. Hubo diferencias estadísticamente significativas en las prevalencias del uso de benzodiazepinas en estado civil $(p<0,001)$ y escolaridad $(p<0,001)$ según la localización rural o urbana del centro de salud.

Aunque el mayor número de prescripciones haya sido en mujeres, no se encontraron 
diferencias significativas al analizar la localización rural o urbana del centro de salud familiar según sexo $(p<0,861)$.

Luego de testear las asociaciones entre las variables de exposición y la ubicación de los centros de salud familiar en las zonas urbana y rural, se consideró la inclusión en el modelo de regresión logística de aquellas variables en las que el valor de $p$ fuera menor que 0,20. La inclusión en modelos de regresión logística mostró una ausencia de asociación entre las variables seleccionadas según los valores de odds ratio. probablemente en función del gran número de participantes sin información en las historias clínicas.

\section{DISCUSIÓN}

Se identificó que, entre los usuarios de benzodiazepinas prescritas por médicos de los centros de atención primaria incluidos en el estudio, hubo un predominio de las mujeres con relación a los varones, como así también del uso en residentes en zonas urbanas, en personas casadas y con baja escolaridad.

Aunque el uso de las benzodiazepinas haya crecido desde la década de 1970 y se hayan consolidado como una medicación efectiva en el tratamiento de trastornos agudos de ansiedad y como coadyuvante en la farmacoterapia de trastornos de humor, insomnio, crisis convulsivas, síndrome de abstinencia alcohólica y otras condiciones relacionadas al sistema nervoso central, su tolerancia y dependencia deben ser consideradas ${ }^{(22)}$.

De acuerdo con la Asociación Médica Brasileña ${ }^{(23)}$, se considera uso prolongado al suministro de medicamentos benzodiazepínicos por un tiempo superior a tres meses. A partir de ese período el paciente ya sería susceptible a la tolerancia y a la dependencia al medicamento. Más allá de los efectos adversos, como somnolencia diurna, cefalea, mareos, fatiga, irritabilidad, ansiedad, náuseas y aumento de crisis de sinusitis( ${ }^{(23)}$, estudios sugieren que pacientes con un uso prolongado de benzodiazepinas presentan mayor probabilidad de manifestar eventos más graves, como la tolerancia, la dependencia y el síndrome de abstinencia, pudiendo presentar también, historia de hipertensión, enfermedad cardíaca isquémica, insuficiencia cardíaca, accidente vascular cerebral, hiperlipidemia, diabetes y enfermedad renal ${ }^{(22,23)}$.

En 2012, un estudio poblacional prospectivo denominado PAQUID, realizado en Francia, relató la asociación entre el uso de benzodiazepinas y el riesgo de desarrollo de demencia, con un $50 \%$ más de posibilidades en los pacientes que usaban esta clase de medicamentos $^{(24)}$. Una investigación más reciente sugiere, además, el aumento del riesgo de mortalidad por todas las causas entre adultos que utilizan benzodiazepinas, incluso por corta duración ${ }^{(25)}$.

Si la dependencia y la tolerancia fisiológica son consecuencias evidentes del suministro prolongado de las benzodiazepinas, cabe aquí resaltar la necesidad de informar tanto al profesional de salud que prescribe la medicación como al paciente que usa el fármaco sobre sus verdaderos efectos a largo plazo. Además, sería importante reconocer los motivos que llevan a la alta tasa de prescripción de estos medicamentos, así como la ausencia de un control sobre la duración del tratamiento, incluso conociendo las complicaciones probadas de su uso.

Cabe resaltar que, en este estudio, la intención fue identificar, en las historias clínicas, el tiempo de uso de las benzodiazepinas; sin embargo, la historia clínica contenía muy poca información respecto del inicio del tratamiento, además de que, en el municipio, fue implementada en soporte electrónico solo seis años antes de la investigación, lo que imposibilitó el acceso a datos históricos. Dado que esta fue una limitación del estudio, se sugiere que se realicen futuros seguimientos, como también cambios en la efectivización de los registros para que esa sea posible realizar un seguimiento de la información.

Orlandi y Noto $^{(26)}$ afirman que el uso indebido y las consiguientes complicaciones desencadenadas por la administración inadecuada de benzodiazepinas se da, en parte, por la falta de información de los pacientes que usan estos medicamentos. A pesar de eso, 
los usuarios exigen que los médicos estén de acuerdo con la prescripción de psicofármacos, sobre todo, cuando usan estas sustancias hace cierto tiempo, y terminan por convencerlos.

Por otro lado, es evidente que, durante la búsqueda de un tratamiento, el paciente deposita una gran confianza en el profesional que lo atiende, por lo que es necesario un empoderamiento del profesional, para que consiga manejar esas situaciones, situación que ha sido descripta como un evento cotidiano, por ejemplo, en $\mathrm{Cuba}^{(20)}$.

Entre los principales motivos que señala la bibliografía del elevado número de prescripciones y el prolongado uso de benzodiazepinas se encuentran la falta de tiempo de los profesionales para cada paciente; poca apropiación de las cuestiones de salud mental; la fragmentación del cuidado; la sobrecarga con otros temas considerados prioritarios, poca inversión en formación específica; la subestimación por parte de los prescriptores de atención primaria en cuanto a la cantidad de pacientes que usan benzodiazepinas o la gravedad de este uso, sin considerar los efectos colaterales de las drogas, o hasta incluso colocando los protocolos propios de la asistencia psiquiátrica como inadecuados a la realidad $^{(20,23)}$. Sin embargo, a pesar de todos estos factores, un estudio realizado en forma conjunta en Cuba y en Brasil mostró que, a pesar de que algunos profesionales reconocieron el uso indiscriminado por parte de los usuarios, no consideraron las propias conductas como decisivas para la consolidación de ese escenario ${ }^{(20)}$.

Por lo tanto, resulta necesario entender el uso de benzodiazepinas como un problema de salud global cuyas complicaciones deben ser consideradas no solo por el médico, sino también por los demás profesionales de los equipos de atención primaria frente al cuidado del paciente con sufrimiento mental.

Además de la prescripción, es necesario pensar en el tiempo de tratamiento adecuado, para lo cual es importante que el profesional tenga un diagnóstico claro para que pueda prescribir el medicamento correcto. Según los datos de la presente investigación, gran parte de las historias clínicas no aportaban información referente al diagnóstico, ni sobre el tiempo que el paciente debería usar aquella medicación o sobre otras medidas más allá de la terapia farmacológica- que el paciente debería ejercer.

Esto mismo se señala en estudios menos recientes. Un estudio escandinavo, por ejemplo, mostró que "de 3.452 prescripciones evaluadas, solo una incluía información sobre la duración del tratamiento"(27). Datos como estos muestran la urgencia de racionar esta clase de medicamentos por parte de los profesionales de salud.

Otro punto relevante se refiere a la prevalencia de la prescripción de benzodiazepinas en sexo femenino y en la población anciana. En nuestro estudio se identificó que la mayoría de las prescripciones fueron a mujeres $(80 \%)$ y franja etaria de 50 a 69 años $(50,7 \%)$. Otros estudios corroboran estos hallazgos, y muestran un uso indebido de benzodiazepinas, por un lado, por parte de ancianos que buscan sobre todo el efecto hipnótico de la medicación y, por otro lado, por individuos de media edad, predominantemente del sexo femenino, que buscan el efecto ansiolítico ${ }^{(26)}$.

Hallazgos similares también fueron relatados por un estudio español del año 2000, que relata una prevalencia mayor del uso de medicamentos de la clase de los benzodiazepinas por mujeres, entre 38 y 70 años, casadas o en relación estable ${ }^{(28)}$. Otro estudio realizado en EEUU, en el año 2016, también identificó un perfil similar, con un uso más prevalente en mujeres de 50 a 64 años, blancas no hispanas, que vivían en la región urbana y contaban con enseñanza media completa ${ }^{(17)}$.

Por un lado, se pueden plantear cuestiones concernientes a la fisiología de la mujer, como también la influencia hormonal sobre la salud mental. Por otro, es evidente que, en el contexto sociocultural, las mujeres son las que más demandan los servicios de salud; sea por una mayor preocupación por un buen estado general, o por su condición social, dado que ellas son más aceptadas al demandar estos servicios.

Además, vale destacar que las cuestiones de género, como las tareas sociales y familiares impuestas a las mujeres, pueden afectar 
de forma negativa la salud mental de la población femenina, y aunque la sobrecarga psicológica sufrida no aflore, aun así, puede no ser soportada ${ }^{(29,30)}$. Ese comportamiento corrobora la prevalencia del sexo femenino en lo referente a las prescripciones realizadas en atención primaria; situación que se refuerza tanto por las atribuciones socioculturales de las mujeres como también por la mayor demanda a los servicios de salud.

Considerando tanto los puntos señalados, como también el perfil de los usuarios que más usan medicamentos de la clase de las benzodiazepinas, se plantea un desafío para las Estrategias de Salud Familiar en lo que respecta a la creación de proyectos terapéuticos singulares que disminuyan la dependencia medicamentosa. Además de considerar el perfil de los usuarios de benzodiazepinas, a fin de proponer terapias asertivas para el cuidado de la salud mental, es necesario también comprender la necesidad de un trabajo multiprofesional, en el que los profesionales de la salud sean corresponsables del tratamiento (o de la falta de este) del usuario en la red de atención a la Salud.

Existen actualmente dos importantes estrategias de salud ya consolidadas en el SUS: los grupos de apoyo y el Programa Nacional de Prácticas Integrales y Complementarias (PNPIC), que incluye acupuntura, homeopatía, plantas medicinales y fitoterapia, termalismo/crenoterapia y la medicina antroposófica ${ }^{(31)}$. Dentro de estas prácticas, la homeopatía - para los episodios de ansiedad en la menopausia ${ }^{(32)}$ - y la acupuntura -para los diversos trastornos de ansiedad ${ }^{(33)}$ - forman parte de la oferta de la red asistencial del municipio de Chapecó.

En ese sentido, son prácticas sustentadas por el SUS ${ }^{(31)}$ que pueden ser complementarias y alternativas al tratamiento farmacológico de pacientes usuarios de benzodiazepinas, ya que según el perfil identificado por este estudio, los pacientes con trastornos de ansiedad y mujeres en período de climaterio y menopausia, son la mayoría de los usuarios tratados con benzodiazepinas en atención primaria.

Ese contexto de alta prescripción de benzodiazepinas y medicamentos en general se pone en discusión a través de los conceptos de medicalización y farmacologización de la vida. En la década de 1970, el concepto de medicalización se tornó relevante como temática social, y sigue vigente hasta la actualidad. Al concebir los problemas sociales como enfermedades que pueden ser tratadas, la medicina opera mecanismos de control social ${ }^{(34)}$. De allí surge el concepto de farmacologización, que cuestiona el consumo de píldoras con la intención de una rápida resolución de los problemas vivenciados, relacionados tanto con el sufrimiento físico como mental ${ }^{(35)}$.

De esa manera, los pacientes pasan a ser rotulados de acuerdo con sus diagnósticos, perdiendo la singularidad ${ }^{(33)}$, restringiéndolos a los síntomas momentáneos. Por eso, se reitera la importancia de conocer el perfil poblacional de los usuarios de los medicamentos más prevalentes, de manera de conocer las condiciones sociales asociadas para establecer un tratamiento multiprofesional, que no se restrinja a la resolución instantánea de síntomas, escenario posible para el universo aquí estudiado.

\section{CONSIDERACIONES FINALES}

Se verificó un elevado número de prescripciones dirigidas a mujeres, adultos y ancianos, que señalan la necesidad de diseñar e implementar acciones que provoquen un cambio de actitud en los gestores y profesionales en cuanto a la atención psicosocial realizada en ámbitos de atención primaria, que revean los procedimientos de prescripción y dispendio de benzodiazepinas. Específicamente, sería oportuno advertir a quienes prescriben los medicamentos sobre la necesidad de aplicar el juicio clínico para alcanzar un diagnóstico adecuado, combinado con un proyecto terapéutico individualizado, que considere los efectos que desencadena la medicación a corto y largo plazo.

Con relación a las cuestiones de género, la dinámica de un mayor uso de los servicios de atención primaria por parte de las mujeres y una mayor medicalización, indica que, 
ante la demanda de un abordaje psicosocial, la prescripción de medicamentos protagonizó el escenario terapéutico.

Por último, cabe destacar la importancia de establecer perfiles poblacionales a fin de promover acciones que se condigan con las reales necesidades en salud pública, y que posibiliten el cuidado especializado y alternativo a la medicación para los pacientes con sufrimiento mental. Actualmente, existen innumerables terapias que pueden ser combinadas, o que pueden hasta incluso sustituir, en algunos casos, el uso de medicamentos. El desarrollo de un efectivo trabajo multiprofesional entre los equipos de Estrategia de Salud Familiar es esencial para que esas terapias sean puestas en prácticas, a fin de pensar la prescripción de medicamentos como una terapia complementaria y no lo contrario.

\section{REFERENCIAS BIBLIOGRÁFICAS}

1. Farinha MG, Braga TBM. Sistema único de saúde e a reforma psiquiátrica: desafios e perspectivas. Phenomenological Studies-Revista da Abordagem Gestáltica. 2018;24(3):366-378.

2. Amarante $P$, Nunes $M O$. A reforma psiquiátrica no SUS e a luta por uma sociedade sem manicômios. Ciência \& Saúde Coletiva. 2018;23(6):2067-2074.

3. Paiva CHA, Teixeira LA. Reforma sanitária e a criação do Sistema Único de Saúde: notas sobre contextos e autores. História, Ciências, Saúde-Manguinhos. $2014 ; 21(1): 15-36$.

4. Sousa PF, Maciel SC, Medeiros KT. Paradigma biomédico x psicossocial: onde são ancoradas as representações sociais acerca do sofrimento psíquico? Temas em Psicologia. 2018;26(2):883-895.

5. Brasil. Lei $n^{\circ} 8.080$, de 19 de setembro de 1990 [Internet]. 1990 [citado 16 may 2018]. Disponible en: https://tinyurl.com/d73vyv.

6. Brasil. Lei $\mathrm{n}^{\circ} 10.216$, de 06 de abril de 2001 [Internet]. 2001 [citado 16 may 2018]. Disponible en: https://tinyurl.com/y2ssrbgx.

7. Guimarães ACO. Uso e abuso dos benzodiazepínicos: revisão bibliográfica para os profissionais de saúde da atenção básica. [Tese Doutorado]. Belo Horizonte: Universidade Federal de Minas Gerais; 2013.

8. Figueiredo MD, Onocko Campos R. Saúde mental na atenção básica à saúde de Campinas, SP: uma rede ou um emaranhado? Ciência \& Saúde Coletiva. 2009;14(1):129-138.

9. Brasil, Ministério da Saúde. Cadernos de Atenção Básica: Saúde mental. No. 34. Brasília: Ministério da Saúde; 2013.

10. Baena VC. Salud mental comunitaria, atención primaria de salud y universidades promotoras de salud en Ecuador. Revista Panamericana de Salud Pública. 2018;42:e162. doi: 10.26633/RPSP.2018.162.
11. Carvalho JLS, Nóbrega MPSS. Práticas integrativas e complementares como recurso de saúde mental na Atenção Básica. Revista Gaúcha de Enfermagem. 2017;38(4):e20170014. doi: 10.1590/1983-1447.2017.04.2017-0014.

12. Anjos MA, Carvalho PAL, Sena ELS, Ribeiro RMC. Acolhimento da pessoa em sofrimento mental na atenção básica para além do encaminhamento. Cadernos Brasileiros de Saúde Mental. 2015;7(16):27-40.

13. Bernik MA, Soares MBM, Soares CN. Benzodiazepínicos padrões de uso, tolerância e dependência. Arquivos de Neuro-Psiquiatria. 1990;48(1):131-137.

14. Katzung BG, Trevor AJ. Farmacologia Básica e Clínica. 13a ed. Porto Alegre: AMGH; 2017.

15. Fiorelli K, Assini FL. A prescrição de benzodiazepínicos no Brasil: uma análise da literatura. ABCS Health Sciences. 2017;42(1):40-44. doi: 10.7322/abcshs.v42i1.948.

16. Maust DT, Lin LA, Blow FC. Benzodiazepine use and misuse among adults in the United States. Psychiatric Services. 2019;70(2):97-106. doi: 10.1176/appi. ps. 201800321.

17. Firmino KF, Abreu MHNG, Perini E, Magalhães SMS. Fatores associados ao uso de benzodiazepínicos no serviço municipal de saúde da cidade de Coronel Fabriciano, Minas Gerais, Brasil. Cadernos de Saúde Pública. 2011;27(6):1223-1232.

18. Nordon DG, Hübner CK. Prescrição de benzodiazepínicos por clínicos gerais. Diagnóstico e Tratamento. 2009;14(2):66-69.

19. Auchewski L, Andreatini R, Galduróz JCF, Lacerda RB. Avaliação da orientação médica sobre os efeitos colaterais de benzodiazepínicos. Revista Brasileira de Psiquiatria. 2004;26(1):24-31.

20. Fegadolli C, Varela NMD, Carlini ELA. Uso e abuso de benzodiazepínicos na atenção primária à saúde: práticas profissionais no Brasil e em Cuba. Cadernos de Saúde Pública. 2019;35(6):e00097718. doi: 10.1590/0102-311x00097718. 
21. Instituto Brasileiro de Geografia e Estatística. Cidades: Chapecó [Internet]. 2019 [citado 2 jul 2020]. Disponible en: https://tinyurl.com/y6gjp68w.

22. Friedman L, Fleming NF, Robert DH, Hyman SE. Source book of substancy abuse and adiction. Baltimore: Willians \& Wilkins; 1996.

23. Associação Médica Brasileira. Abuso e dependência de benzodiazepínicos [Internet]. 2013 [citado 23 jul 2020]. Disponible en: https://tinyurl.com/y2qdgsl6.

24. Billioti de Gage S, Bégaud B, Bazin F, Verdoux H, Dartigues JF, Pérès K, Kurth $T$, Pariente A. Benzodiazepine use and risk of dementia: prospective population based study. BMJ. 2012;345(6):e6231.

25. Patorno E, Glynn RJ, Levin R, Lee MP, Huybrechts KF. Benzodiazepines and risk of all cause mortality in adults: cohort study. BMJ. 2017;358:j2941. doi: 10.1136/bmj.j2941.

26. Orland P, Noto AR. Uso indevido de benzodiazepínicos: um estudo com informantes-chave no município de São Paulo. Revista Latino-Americana de Enfermagem. 2005;13(Spec):896-902.

27. Straand J, Rokstad K. General practitioners' prescribing patterns of benzodiazepine hypnotics: are elderly patients at particular risk for overprescribing? A report from the Møre \& Romsdal Prescription Study. Scandinavian Journal of Primary Health Care. 1997;15(1):16-21. doi: 10.3109/02813439709043423.

28. Escrivá Ferrairó R, Pérez Díez A, Lumbreras García C, Molina Parísa J, Sanz Cuestad T, Corral Sánchez MA. Prescripción de benzodiacepinas en un centro de salud: prevalencia, cómo es su consumo y características del consumidor. Atención Primaria. 2000;25(2):107-110. doi: 10.1016/S0212-6567(00)78472-1.

29. Bezerra DS, Bonzi ARB, Silva GR, Lima AKBS. Mulheres e o uso de benzodiazepínicos: uma revisão integrativa. Temas em Saúde. 2018;18(2):204-2015.

30. Azevedo AJP, Araujo AA, Ferreira MAF. Consumo de ansiolíticos benzodiazepínicos: uma correlação entre dados do SNGPC e indicadores sociodemográficos nas capitais brasileiras. Ciência \& Saúde Coletiva. 2016;21(1):83-90.

31. Brasil, Ministério da Saúde. Política Nacional de Práticas Integrativas e Complementares no SUS: Atitude de ampliação de acesso. Brasília: Ministério da Saúde; 2006.

32. Martins PM. A homeopatia como tratamento alternativo no climatério. [Internet]. São Paulo: Associação Paulista de Homeopatia; 2014 [citado 16 may 2018]. Disponible en: https://tinyurl.com/yy2oz3kc.

33. Silva ALP. O Tratamento da ansiedade por intermédio da acupuntura: um estudo de caso. Psicologia Ciência e Profissão. 2010;30(1):200-211.

34. Carvalho SR, Rodrigues CO, Costa FD, Andrade HS. Medicalização: uma crítica (im)pertinente? Introdução. Physis: Revista de Saúde Coletiva. 2015;25(4):12511269.

35. Galindo DCG, Lemos FCS, Vilela R, Garcia B. Medicalização e governo da vida e subjetividades: o mercado da saúde. Estudos e Pesquisas em Psicologia. 2016;16(2):346-365.

FORMA DE CITAR

Oliveira-Friestino JK, Luzardo AR, Gralak EZ, Gass LS, Guimarães PGZ, Barbato PR. El perfil de usuarios de benzodiazepinas en servicios de atención primaria de la salud de la ciudad de Chapecó, Santa Catarina, Brasil. Salud Colectiva. 2020;16:e2495. doi: 10.18294/sc.2020.2495.

Recibido: 2 sep 2019 | Versión final: 26 jul 2020 | Aprobado: 6 ago 2020 | Publicado en línea: 3 sep 2020

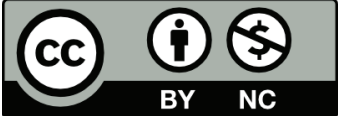

Esta obra está bajo una licencia de Creative Commons Reconocimiento-NoComercial 4.0 Internacional. Reconocimiento - Permite copiar, distribuir y comunicar públicamente la obra. A cambio, se debe reconocer y citar al autor original. No Comercial - Esta obra no puede ser utilizada con finalidades comerciales, a menos que se obtenga el permiso. 